\title{
Microwave Signal Processing over Multicore Fiber
}

\author{
Sergi García, David Barrera, Javier Hervás, Salvador Sales (1D) and Ivana Gasulla * \\ ITEAM Research Institute, Universitat Politècnica de València, 46022 Valencia, Spain; \\ sergarc3@iteam.upv.es (S.G.); dabarvi@iteam.upv.es (D.B.); jaherpe2@iteam.upv.es (J.H.); \\ ssales@dcom.upv.es (S.S.) \\ * Correspondence: ivgames@iteam.upv.es
}

Received: 7 November 2017; Accepted: 3 December 2017; Published: 8 December 2017

\begin{abstract}
We review the introduction of the space dimension into fiber-based technologies to implement compact and versatile signal processing solutions for microwave and millimeter wave signals. Built upon multicore fiber links and devices, this approach allows the realization of fiber-distributed signal processing in the context of fiber-wireless communications, providing both radiofrequency access distribution and signal processing in the same fiber medium. We present different space-division multiplexing architectures to implement tunable true time delay lines that can be applied to a variety of microwave photonics functionalities, such as signal filtering, radio beamsteering in phased array antennas or optoelectronic oscillation. In particular, this paper gathers our latest work on the following multicore fiber technologies: dispersion-engineered heterogeneous multicore fiber links for distributed tunable true time delay line operation; multicavity devices built upon the selective inscription of gratings in homogenous multicore fibers for compact true time delay line operation; and multicavity optoelectronic oscillation over both homogeneous and heterogeneous multicore fibers.
\end{abstract}

Keywords: microwave photonics; space-division multiplexing; radiofrequency signal processing; multicore fibers; fiber Bragg gratings; optoelectronic oscillators; true time delay lines

\section{Introduction}

The addition of the spatial dimension to the portfolio of optical multiplexing technologies has been welcomed by the optical communications community as a promising solution to the capacity saturation of conventional single-mode fibers (SMFs) [1]. Space-Division Multiplexing (SDM) aims to increase the transmission capacity over a fixed bandwidth by increasing the number of light paths that are transmitted within the same fiber cladding. Different SDM technologies have been investigated over the last few years including either multicore fibers (MCFs) [2-26], few-mode fibers (FMFs) [27] or a combination of both [28]. In the case of MCFs, we can transmit the multiplex of data signals along the different cores that comprise the fiber while keeping a low level of crosstalk. Most of the research activity on MCF transmission has employed the so-called homogeneous multicore fiber, where $N$ identical cores are confined inside a single cladding with an outer diameter ranging from 125 to $260 \mu \mathrm{m}$. The highest capacities demonstrated so far have been realized by means of uncoupled propagation featuring crosstalk values between -72 and $-22 \mathrm{~dB} / 100 \mathrm{~km}$ [3]. Here, the use of trench-assisted refractive index profiles plays an important role. Thanks to a better confinement of the optical field, this technique makes the crosstalk insensitive to the fiber bending radius increase, allowing very high capacities as, for instance, the $2.15 \mathrm{~Pb} / \mathrm{s}$ reached in a 22-core fiber in [2].

On the other hand, heterogeneous MCFs were proposed in 2009 to increase the density of cores as compared to homogeneous MCFs. They are composed of non-identical cores characterized by different propagation constants, arranged so that the crosstalk between any pair of neighboring cores is reduced as the phase matching condition is prevented. This crosstalk reduction allows reducing 
the core pitch of the fiber and thus increase the number of cores per cross-sectional fiber area. Initial designs of heterogeneous 19-core fibers obtained a core pitch reduction down to $23 \mu \mathrm{m}$ in a $125-\mu \mathrm{m}$ cladding diameter by accommodating three different core compositions [9]. Further progress in crosstalk management has also incorporated trench-assisted heterogeneous configurations $[5,10,20]$. This led to larger core multiplicities as, for instance, the 30-core fiber designed with four different types of trench-assisted cores in a cladding diameter of $228 \mu \mathrm{m}$ [26]; and the recent 37-core fiber fabricated by Sasaki et al. in 2017 using three types of trench-assisted cores arranged in a 248-um cladding diameter [25]. Recent advances incorporating few-mode transmission in each core have achieved a record spatial channel over 100, for example by combining 36 heterogeneous cores and three-mode transmission in a 306- $\mu \mathrm{m}$ cladding diameter [28].

In general, with the premise of reducing size, weight, power consumption and cost, the field of Microwave Photonics (MWP) moves towards the integration of the different photonic processing and distribution elements. In this sense, photonic integrated circuits (where processing components/subsystems are integrated in monolithic or hybrid photonic circuits) could be considered as a "vertical integration", while the use of SDM fiber-distributed technologies could be considered as a "horizontal integration", that is, integration in a distributed way. The synergy between both disciplines will be key for the future of Microwave Photonics, where SDM fiber technologies will benefit from the implementation of integrated transceivers and interconnection elements. Up to date, research has focused on the implementation of mode multiplexers/demultiplexers for FMF transmission $[29,30]$ and fan-in/fan-out devices for MCF transmission [29,31], including optical MIMO processing [29] as well as MCF switches towards optical add/drop multiplexers [31]. One of the main challenges here is to couple light from/to an SDM fiber efficiently to/from the facet of a silicon photonic waveguide. Various solutions have been published, including one-dimensional grating couplers arranged in a triangular lattice to match the core distribution of a MCF [29], planar two-dimensional grating couplers driven in anti-phase for mode mux/demux [30], and photonic wire bonding [32].

Even though MCFs were initially envisioned in the context of core and metro optical networks, they can also be applied to a wide range of application fields including radio access networks and multiple antenna connectivity [6,33,34], multi-parameter optical fiber sensing [35] and radiofrequency (RF) signal processing [36-39]. For instance, Diamandi et al. have recently demonstrated that opto-mechanical intercore crosstalk in MCFs can be very useful for applications such as multicore fiber lasers, parametric amplification in SDM networks for MCFs, sensing or optoelectronic oscillators (OEOs) [15]. In particular, Microwave Photonics signal processing applications, such as microwave signal filtering, beamsteering in phased array antennas and arbitrary waveform generation, can benefit greatly from the use of MCFs in terms of compactness and weight, as well as in terms of performance stability and versatility. The current trend in MWP is to dedicate a given fiber-based device or photonic integrated circuit to process the RF signal, while the distribution of the signal is performed by an external optical fiber link. We propose a new MWP approach where we can implement the required signal processing functionalities while we distribute the RF signal to the end user (wireless base station, indoor antenna, radar antenna, etc.). This leads to what we call "fiber-distributed signal processing", a concept with great potential regarding converged fiber-wireless telecommunications scenarios, as those envisioned for 5G systems and the Internet of Things. A true time delay line (TTDL) can be considered the key building block of radiofrequency signal processing [36-47]. This photonic subsystem provides a frequency independent and tunable group delay for the RF signal within a given frequency range. A variety of photonic technology approaches have been reported for the implementation of optical TTDLs over the past 30 years, using either fiber-based systems or photonic integrated circuits. Configurations built upon single-core SMFs featured operation bandwidths ranging from 0.1 up to $40 \mathrm{GHz}$ and RF delays ranging from 0.4 up to $8 \mathrm{~ns}$. These configurations include the use of switched fiber architectures and dispersive fibers [40], the inscription of different Fiber Bragg Grating (FBG) devices [41-43] as well as the exploitation of nonlinearities such as Stimulated Brillouin Scattering [44]. On the other hand, integrated photonics 
technologies have demonstrated operation bandwidths from 2 to $50 \mathrm{GHz}$ and RF delays from 40 to 140 ps. Significant examples include ring cavities in Silicon on Insulator [45], racetrack resonators in Silicon Nitride [45] as well as photonic crystal structures [46] and semiconductor amplifiers [47] based on Indium Phosphide.

Figure 1 shows the general idea that underlies the TTDL built upon a multicore fiber. The goal here is to obtain at the output of the fiber link or device a given set of time-delayed samples of the modulating RF signal that was injected at the input. This set of replicas must be characterized by a constant basic differential delay $\Delta \tau$ experienced between adjacent samples. We identify this configuration as a 1D (one-dimensional) TTDL since all the samples are given by exploiting the spatial diversity (or core diversity) behavior of the MCF. However, this approach potentially offers not only 1D, but also 2D (two-dimensional) operation if we incorporate the optical wavelength diversity provided, for instance, by an array of lasers emitting at different optical wavelengths. This very general idea will adapt properly to the particular type of MCF technology employed. In the case of a heterogeneous MCF, we can design each core with a different refractive index profile as to provide a different group delay [48-50]. In the case of homogeneous MCFs, where all the cores are in principle identical, we must incorporate dispersive optical elements in each one of the cores [51].

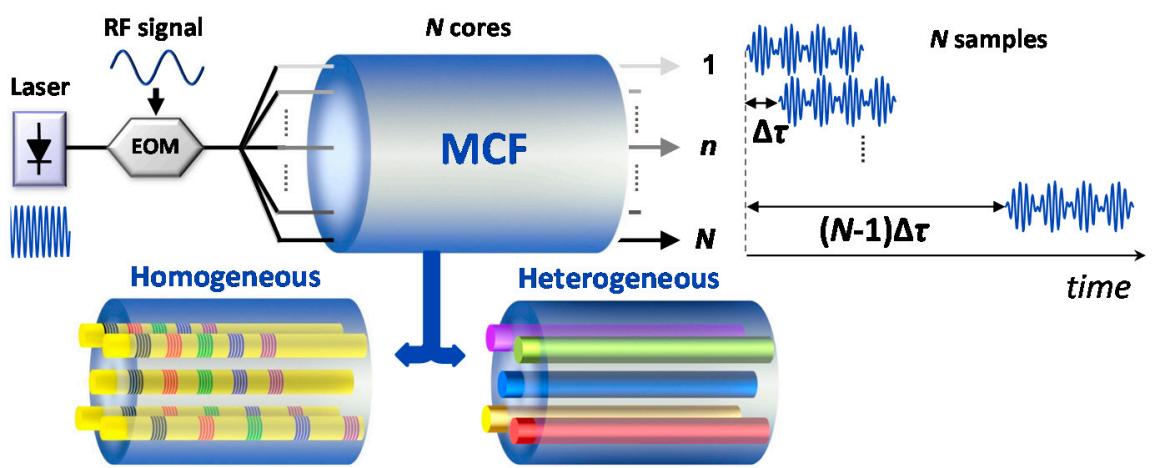

Figure 1. General concept underlying the sampled true time delay line built upon a multicore fiber.

We gathered in this paper our latest work on the area of radiofrequency signal processing using different space-division multiplexing technologies. First, we addressed how to design dispersion-engineered heterogeneous MCFs to act as distributed and tunable true time delay lines. Then, we reviewed our work on the fabrication of compact TTDL devices based on the inscription of grating elements along the cores of a homogeneous MCF. Finally, optoelectronic oscillation is presented as one of the MWP applications that benefits from the fiber integration offered by SDM.

\section{Sampled Delay Line Operation over a Heterogeneous MCF Link}

Most of the research activity in heterogeneous MCFs is focused on high-capacity digital communications. While keeping equal propagation characteristics (in particular the value of the chromatic dispersion parameter D) in all the cores is important in digital SDM applications, for the implementation of tunable TTDLs it is important to have different values at a fixed wavelength. Therefore, a new procedure for designing heterogeneous MCFs has to be developed where, while keeping the advantages in terms of crosstalk and immunity to bends, one can have the flexibility of tailoring the chromatic dispersion parameter of each individual core. With that aim in view, we have proposed and evaluated seven-sample tunable TTDLs based on different designs of heterogeneous seven-core fibers [48-50].

The design of heterogeneous MCFs to operate as group-index-variable delay lines implies that each core features an independent group delay with a linear dependence on the optical wavelength. 
The group delay $\tau_{g, n}(\lambda)$ of a particular core $n$ can be expanded in its 2 nd-order Taylor series' around an anchor wavelength $\lambda_{0}$ as

$$
\tau_{g, n}(\lambda)=\tau_{g, n}\left(\lambda_{0}\right)+D_{n} \cdot\left(\lambda-\lambda_{0}\right)+\frac{1}{2} \cdot S_{n} \cdot\left(\lambda-\lambda_{0}\right)^{2},
$$

where $D_{n}$ is the chromatic dispersion and $S_{n}$ is the dispersion slope of core $n$ at $\lambda_{0}$. For proper TTDL operability, $D_{n}$ must increase linearly with the core number $n$ while keeping a linear behavior of the group delay with the optical wavelength. If we force all cores to share the same group delay value at $\lambda_{0}$, we can control the basic differential delay between samples, $\tau_{g, n+1}(\lambda)-\tau_{g, n}(\lambda)$, to provide continuous tunability from 0 to $10 \mathrm{~s}$ (or even $100 \mathrm{~s}$ ) of $\mathrm{ps} / \mathrm{km}$. This gives us the possibility of implementing fiber-distributed signal processing links with lengths up to a few kilometers.

We must therefore customize the refractive index profile of each single core to provide the desired spectral delay characteristics. The use of trench-assisted cores improves not only the intercore crosstalk robustness, but also offers more design versatility since more design parameters are involved [49]. Figure 2a shows the schematic of the trench-assisted step-index refractive index profile, where $a_{1}$ is the core radius, $a_{2}$ is the core-to-trench distance, $w$ is the trench width, $\Delta_{1}$ is the core-to-cladding relative index difference, and $\Delta_{2}$ is the cladding-to-trench relative index difference. By suitable modifications of these parameters, we can obtain the required linearly incremental chromatic dispersion values and the common group delay at the anchor wavelength. In [50], we found an optimum TTDL design in terms of both time delay tunability and crosstalk for a seven-core fiber with trench-assisted step-index core profiles. Figure $2 \mathrm{~b}, \mathrm{c}$ represents the computed group delay and dispersion for each core as a function of the core radius for all the cores. As shown, by properly adjusting the design parameters at the anchor wavelength $\lambda_{0}=1.55 \mu \mathrm{m}$, we obtain: (1) identical group delays at $\lambda_{0}$; and (2) a set of dispersion values ranging from 14.75 to $20.75 \mathrm{ps} /(\mathrm{km} \cdot \mathrm{nm})$ with an incremental dispersion of $1 \mathrm{ps} /(\mathrm{km} \cdot \mathrm{nm})$.

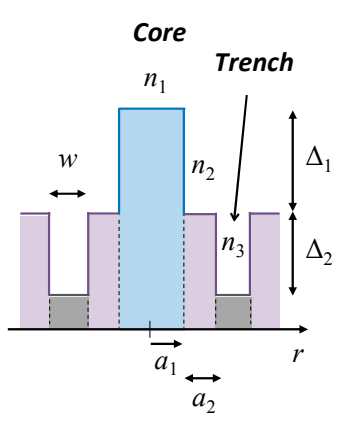

(a)

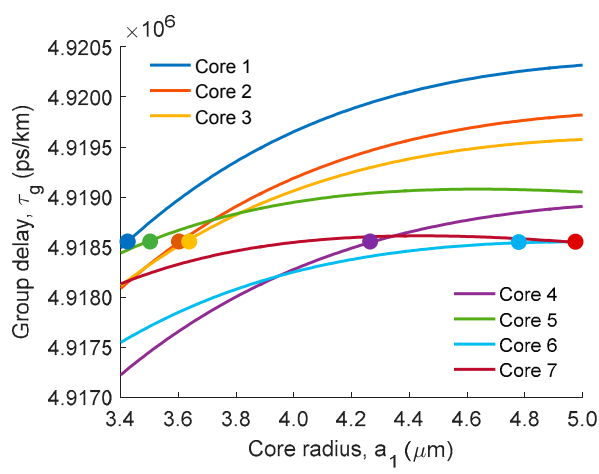

(b)

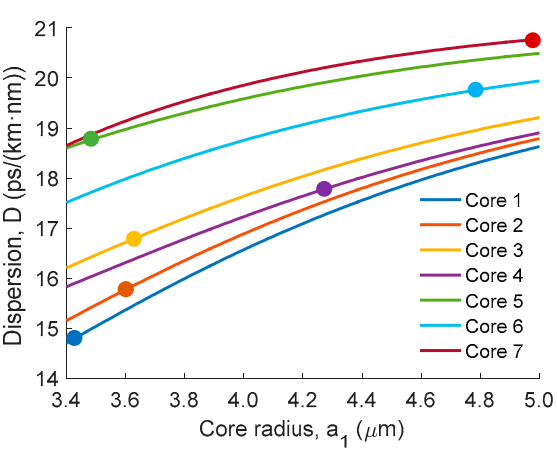

(c)

Figure 2. (a) Refractive index profile of the fiber cores; (b) group delay; and (c) chromatic dispersion of each core as a function of the core radius. Each filled circle represents the selected core radius and the corresponding group delay and chromatic dispersion values.

As required in heterogeneous MCF links, we must assure a low level of intercore crosstalk as well as robustness against fiber curvatures. At a first stage of the design process, we focused mainly on reducing the crosstalk, so we aimed to reduce the radius of the cores as to minimize the coupling coefficient $[3,4]$. Apart from the expected intercore crosstalk minimization, we found that the higher-order dispersion (i.e., the dispersion slope) induces serious detrimental effects that can degrade the performance of the delay line. Thus, we decided to move to higher core radii while keeping good crosstalk responses and preserving the linear behavior of the group delay with the optical wavelength in a wide range. The performance of the delay line in terms of intercore crosstalk and robustness against curvatures can be controlled by properly adjusting the effective index of the cores. One of the major detrimental effects that can degrade the performance of heterogeneous MCFs is the crosstalk dependence on the phase-matching condition between adjacent cores when the fiber 
is bent [3-5]. The threshold bending radius, $R_{p k}$, which is the maximum curvature that can induce a phase matching between any pair of adjacent cores, can be expressed as [5],

$$
R_{p k}=\Lambda \cdot n_{e f f, n} /\left|n_{e f f, n}-n_{e f f, m}\right|,
$$

where $\Lambda$ is the core pitch and $n_{\text {eff }, n}$ is the effective index of core $n$. To minimize the threshold bending radius as much as possible, we designed the core refractive index profiles and placed the cores inside the fiber cross section as to maximize the difference in the effective indices of adjacent cores Figure $3 \mathrm{a}$ represents the schematic cross section of the fiber. The core pitch was set to $35 \mu \mathrm{m}$ and the cladding diameter to $125 \mu \mathrm{m}$. The selected values for the design parameters together with the computed group delays, dispersions and effective indices of each core are summarized in Table 1 . We simulated the worst-case crosstalk versus bending radius for a 10-km MCF link. We see in Figure $3 \mathrm{~b}$ that the threshold bending radius is located at $103 \mathrm{~mm}$, as expected from Equation (2), and the crosstalk level above the phase matching region is kept below $-90 \mathrm{~dB}$.

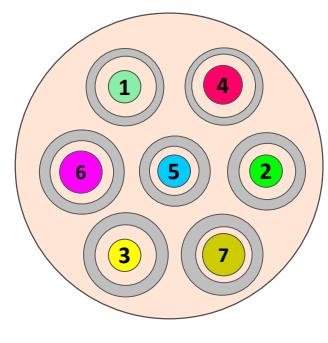

(a)

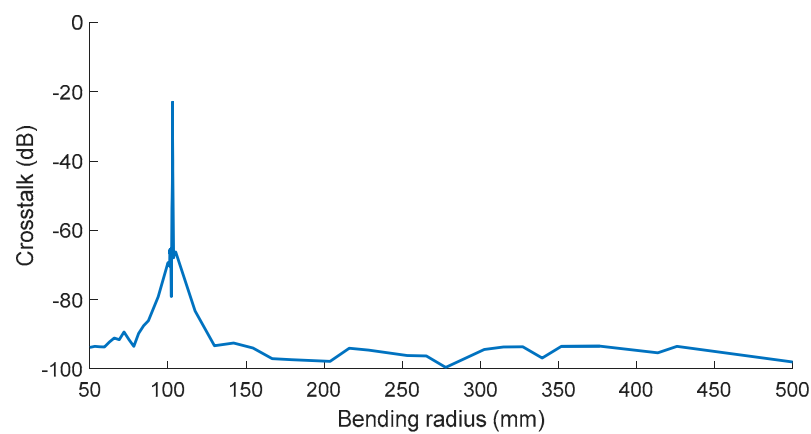

(b)

Figure 3. (a) Schematic cross section of the designed multicore fiber; and (b) computed worst-case crosstalk as a function of the bending radius [50].

Table 1. Core design parameters and computed fiber characteristics of the designed MCF [50].

\begin{tabular}{cccccccc}
\hline Core & $\boldsymbol{a}_{\mathbf{1}}$ & $\boldsymbol{a}_{\mathbf{2}}$ & $\boldsymbol{w}$ & $\boldsymbol{\Delta}_{\mathbf{1}} \mathbf{( \% )}$ & $\boldsymbol{\tau}_{\boldsymbol{g}}(\mathbf{p s} / \mathbf{m})$ & $\boldsymbol{D}(\mathbf{p s} /(\mathbf{k m} \cdot \mathbf{n m}))$ & $\boldsymbol{n}_{\text {eff }}$ \\
\hline 1 & 3.42 & 5.48 & 3.02 & 0.3864 & 4918.543 & 14.75 & 1.4534 \\
2 & 3.60 & 5.03 & 2.61 & 0.3762 & 4918.543 & 15.75 & 1.4535 \\
3 & 3.62 & 4.35 & 3.32 & 0.3690 & 4918.543 & 16.75 & 1.4534 \\
4 & 4.26 & 4.92 & 4.67 & 0.3588 & 4918.543 & 17.75 & 1.4539 \\
5 & 3.49 & 2.81 & 5.41 & 0.3476 & 4918.543 & 18.75 & 1.4529 \\
6 & 4.79 & 3.35 & 3.32 & 0.3435 & 4918.543 & 19.75 & 1.4540 \\
7 & 4.98 & 2.42 & 4.05 & 0.3333 & 4918.543 & 20.75 & 1.4540 \\
\hline
\end{tabular}

The designed seven-core fiber offers a linear and incremental behavior of the group delay of each core as a function of the optical wavelength in a wide wavelength range, as shown in Figure 4a. This allows us to implement tunable TTDLs that can be used as the basic element to perform multiple MWP applications. As a proof of concept, we evaluated the performance of this delay line when it is applied to implement typical signal processing tasks, such as microwave signal filtering and radio beamsteering for phased array antennas. Figure $4 \mathrm{~b}$ shows the computed responses of the corresponding beamforming (upper part) and filtering (lower part) functionalities for a 10-km link. This delay line allows us the possibility of continuously tuning the free spectral range (FSR) of the filter and the antenna beam direction by changing the operation wavelength of the laser. For instance, we see how changing the operation wavelength from 1560 up to $1575 \mathrm{~nm}$ induces a reduction on the FSR from 10 to $4 \mathrm{GHz}$ and a variation on the beam direction from 180 to $90^{\circ}$ without any significant degradation due to higher-order dispersion effects. 


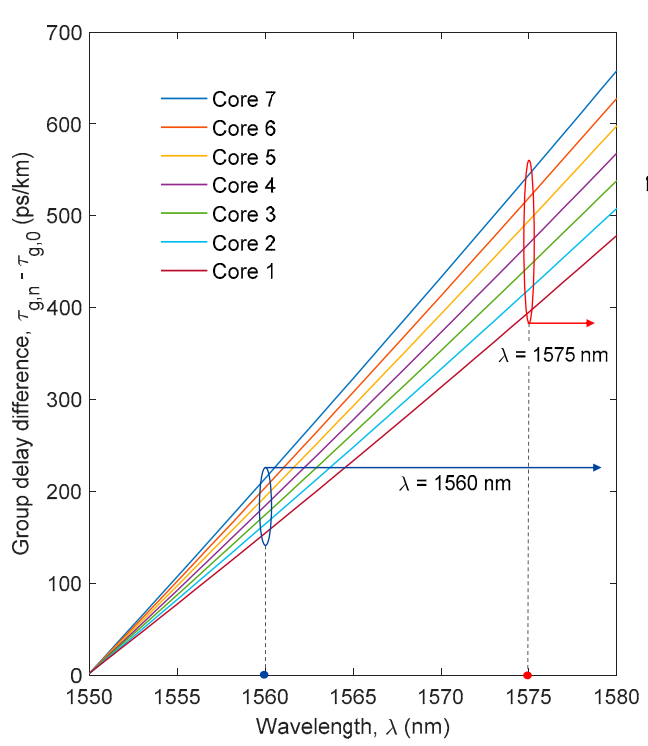

(a)

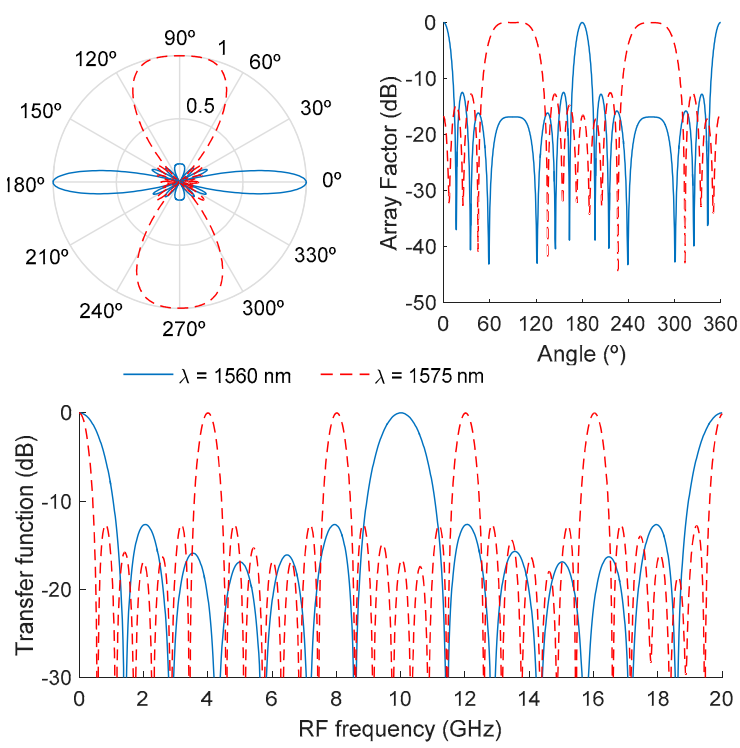

(b)

Figure 4. (a) Computed group delay of each core as a function of the optical wavelength for the designed multicore fiber; and (b) computed array factor as a function of the beam pointing angle (upper part) and computed transfer function as a function of the radio frequency (lower part) for the 10-km link of the designed fiber and an operation wavelength of $1560 \mathrm{~nm}$ (blue solid lines) and $1575 \mathrm{~nm}$ (red dashed lines).

Despite the expected potential of these fibers, we must take into account that their performance in terms of TTDL operability could be degraded due to inaccuracies in the fabrication process. Since the fabrication tolerances for MCFs are currently in the order of $\pm 0.1 \mu \mathrm{m}$ for the core radius, important discrepancies on the designed dispersion and group delay values may occur. In particular, we could expect a variation on the designed values up to several $\mathrm{ps} / \mathrm{km}(\sim 10 \mathrm{ps} / \mathrm{km})$ for the group delay and more than $0.2 \mathrm{ps} / \mathrm{km} / \mathrm{nm}$ for the dispersion. Actually, the fabrication process would involve several fabrication and re-design stages to allow the manufacturers to properly optimize the process with the feedback returned from our evaluations. To minimize possible detrimental effects, we have obtained a robust-to-fabrication-tolerances design that trades part of its delay line potential to overcome the fabrication discrepancies. Our main strategy here is to avoid the matching point of the group delays at the anchor wavelength, forcing the group delays at that wavelength $\tau_{g, n}\left(\lambda_{0}\right)$ to follow a similar incremental law as the dispersion does (see Table 1). With this solution, we expect a maximum relative error on the delay line performance lower than $\pm 10 \%$. Nevertheless, this robustness will lead to a tradeoff with the frequency tunability of the delay line, so we may expect a reduction on its tunability to a 10-GHz range, while the original design is expected to cover from a few $\mathrm{GHz}$ up to the mm-wave band.

\section{Sampled Delay Line Operation over a Homogeneous MCF Multicavity Device}

Most of the research activity on SDM has employed the so-called homogeneous multicore fiber, where all the cores are in principle identical and, thus, share the same propagation characteristics, i.e., the same group delay $\tau$ and chromatic dispersion parameter $D$. The implementation of the tunable true time delay line that we propose will however require different values of these parameters at each core. Therefore, a novel approach for designing true time delay lines based on commercial homogeneous MCFs should be accomplished through the in-line incorporation of additional dispersive optical elements in each core, while preserving the crosstalk levels. In this case, one should design a multicavity device where the different cavities are produced by the inscription of individual FBGs at selective positions along the cores. 
Optical devices built upon the inscription of FBGs in single-core single-mode fibers have been widely investigated as dispersive 1D (1-dimensional) sampled delay lines [43], where the use of the optical wavelength diversity allows the tunability of the basic differential delay $\Delta \tau$. If we incorporate the spatial or core diversity, we obtain a 2D delay line that offers a higher degree of flexibility, versatility and compactness. Actually, previous work on FBG inscription in MCFs has focused mainly on the simultaneous writing of the same grating (i.e., placed at the same position) in all the cores, mainly for sensing and astrophotonics [35,52]. To inscribe individual gratings in a selective way, we developed the first fabrication method to inscribe high-quality gratings located in arbitrary longitudinal positions along the selected fiber core [51]. Figure 5 shows the scheme of the FBG writing setup that is based on the moving phase mask technique. First, the laser beam is conditioned by a mirror that is mounted in a piezo-electric transducer that can induce a beam vertical deflection and then by two cylindrical lenses that allow the control of both beam height and width. The beam width and the distance between the fiber and the phase mask were adjusted to control the interference pattern created after the phase mask. For a phase mask with a period of $1070 \mathrm{~nm}$, the maximum width that allows inscription in a single outer core is approximately $23 \mu \mathrm{m}$. On the other hand, the beam height, which is determined by the size of the cores and their arrangement in the fiber cross section, was adjusted within the range from 30 to $50 \mu \mathrm{m}$.

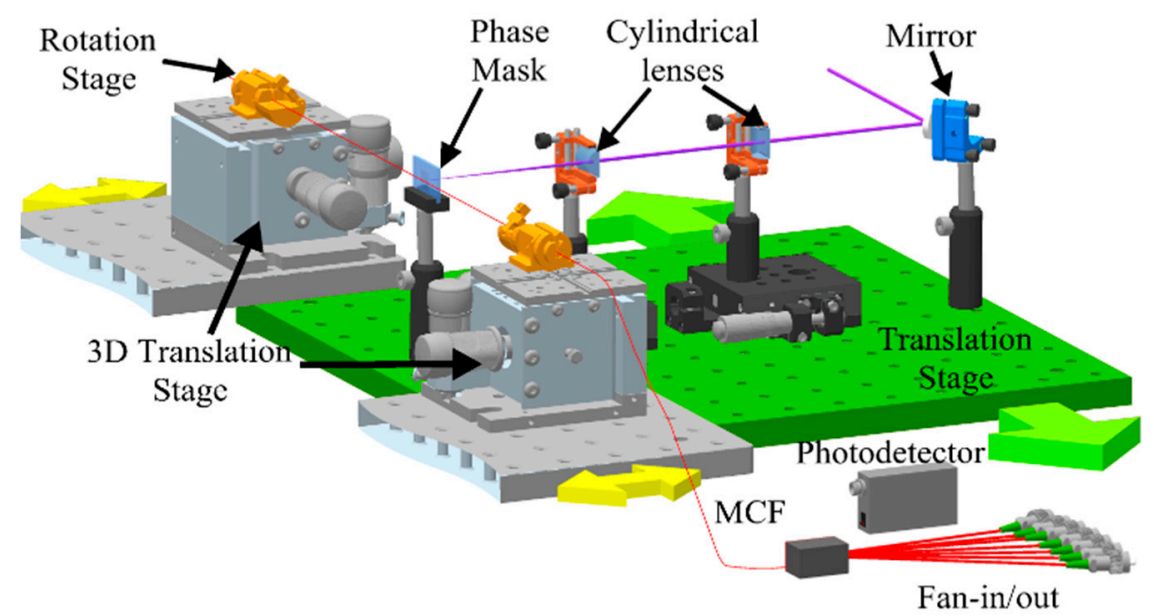

Figure 5. General scheme of the fiber Bragg grating inscription setup [51].

By slightly modifying the above technique, we have fabricated different multicavity TTDL devices, either inscribing the same FBG in groups of cores or in individual cores [51]. Figure 6a shows, as an example, the scheme of a multicavity device that is based on the selective inscription of FBGs in three outer cores, identified as 4, 5 and 6 . The MCF is a commercial seven-core fiber characterized by a cladding diameter of $125 \mu \mathrm{m}$ and a core pitch of $35 \mu \mathrm{m}$. Each one of these cores comprises an array of three uniform gratings, being each one centered at a different wavelength: $\lambda_{1}=1537.07 \mathrm{~nm}$, $\lambda_{2}=1541.51 \mathrm{~nm}$ and $\lambda_{3}=1546.26 \mathrm{~nm}$. To assure that the delay line operates in wavelength diversity, we have written the arrays to feature incremental distances between FBGs when going from core to core, i.e., 20-mm, 21-mm and 22-mm distances, respectively, within cores 6, 5 and 4 . On the other hand, the spatial or core diversity arises from the incremental displacement between FBGs centered at the same wavelength but inscribed in adjacent cores, i.e., 6- $\mathrm{mm}, 7-\mathrm{mm}$ and $8-\mathrm{mm}$ displacements, respectively, for the wavelengths $\lambda_{1}, \lambda_{2}$ and $\lambda_{3}$. As appreciated from the optical spectra in reflection gathered in Figure $6 b$, all the FBGs present similar levels of strength with a maximum difference of $3 \mathrm{~dB}$. 

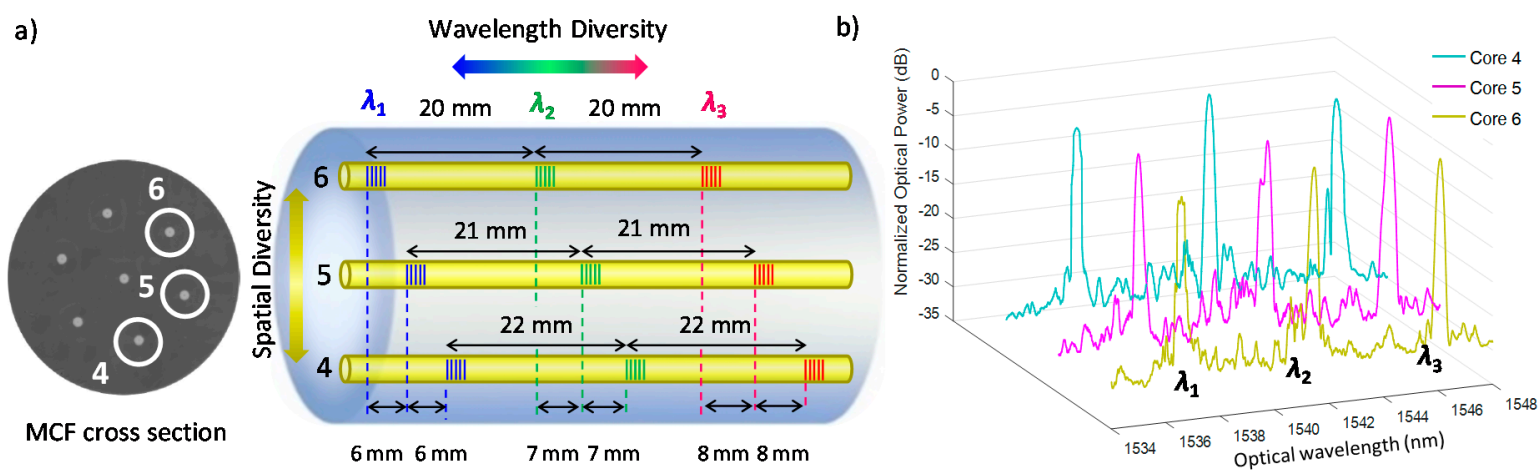

Figure 6. (a) Schematic of the multicavity device built by selectively inscribing fiber Bragg gratings in three of the outer cores of the commercial homogeneous seven-core fiber; and (b) corresponding measured optical spectrum in reflection [51].

The performance of this multicavity delay line device was evaluated in the context of microwave signal filtering [36]. To achieve the filtering effect, we combined and photodetected all the delayed RF signal samples coming from the MCF device output. Figure 7a illustrates the experimental setup where we used either an array of three low-linewidth lasers or a broadband optical source followed by a 2-nm-bandwidth filter. Actually, the broadband source is required to avoid coherent interference when we detect together the samples coming from different cores with a differential delay lower than the coherence time of the low-linewidth laser. We see in Figure $7 \mathrm{~b}$ the measured radiofrequency responses for the filters obtained when we operate in wavelength diversity, i.e., gathering the delayed samples coming from a given single core. By changing from core to core (core 4, 5 or 6 ), we can reconfigure the filter, varying the FSR from 4.45 up to $4.97 \mathrm{GHz}$. The same device can operate using the spatial diversity provided by the cores if we detect the delayed samples that come from different cores but share to the same wavelength. Figure $7 \mathrm{c}$ shows the measured responses given by each of the three input optical wavelengths, where the FSR varies from 12.50 up to $17.76 \mathrm{GHz}$. These experimental results are actually in very good agreement with the theoretical ones, with small discrepancies in the basic differential delays that may be caused by discrepancies between the theoretical and actual values of the core refractive indices and small imbalances in the reflectivity strength of the FBGs.
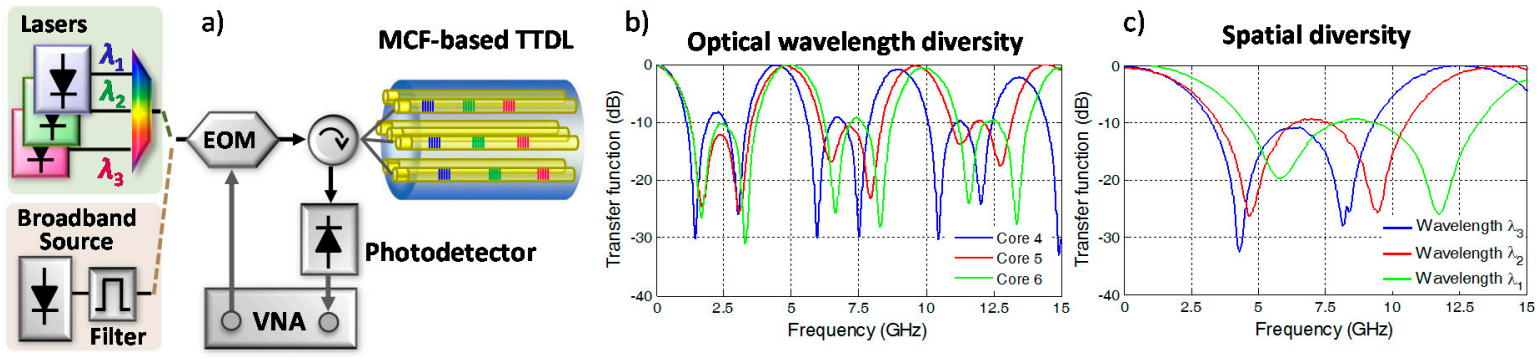

Figure 7. (a) Microwave signal fileting experimental setup; and measured radiofrequency responses operating in: (b) wavelength diversity (for each one of the radiated cores); and (c) spatial diversity (for each one of the optical wavelengths) [51].

\section{Multiloop Optoelectronic Oscillation over a Multicore Fiber}

Multicavity or multiloop optoelectronic oscillators [53-57] can benefit from a reduction of size and weight if the different cores of a multicore fiber act as the required oscillation cavities. In addition, since all the cavities are hosted within the same cladding, this approach provides a fiber integrated medium for enhancing the OEO relative stability against environmental and mechanical fluctuations. 
Introduced by Yao and Maleki in 1996, first in a single-cavity configuration [58], OEOs can generate $\mathrm{RF}$ signals with a low linewidth in a feedback-loop structure along a broad frequency range. A high level of spectral purity in OEOs will require a long fiber loop, what translates into a large number of oscillation modes that are separated by a small frequency period. Therefore, very selective RF filters will be required to select only a single oscillating mode. In 2000, dual-cavity OEOs were conceived to alleviate this narrowband RF filter requirement [53]. In essence, a short loop provides the required spectral separation between adjacent oscillating modes while a long loop is responsible for the spectral purity. The number of loops can as well be generalized to an arbitrary number [54-57,59]. In concrete, the work reported in [54] experimentally demonstrated three-cavity OEOs by using three different single-mode fiber links whose lengths were multiple of a given reference value.

The incorporation of MCFs as the hosting medium for all the cavities was first proposed and theoretically evaluated in [55] considering both homogeneous and heterogeneous fiber configurations. There, we show how homogeneous MCFs allow for two different OEO architectures, highly unbalanced dual-cavity operation (the cavity lengths are a multiple of a reference value) and multi-cavity vernier operation (the cavity lengths are slightly different), where moderate and long cavity lengths $(>2 \mathrm{~m})$ are compatible with a high-spectral purity and multi-GHz oscillation mode spectral separation. The experimental demonstration of both multicavity architectures was reported for the first time in [59] over a 20-m-long commercial homogeneous MCF link. This MCF comprises seven cores and is characterized by a cladding diameter of $125 \mu \mathrm{m}$, a core separation of $35 \mu \mathrm{m}$ and a core mode field diameter of $6.4 \mu \mathrm{m}$. As an example, we gather here the main experimental results obtained for a three-cavity multicavity Vernier optoelectronic oscillator. Figure 8 shows the experimental setup where three cores of the MCF behave as the three cavities of the oscillator. For simplicity, we used a 3-m long external SMF to produce the required incremental delay between cavities to achieve the Vernier effect, and variable delay lines (VDLs) to adjust finely the length difference between cavities. Once the signals are photodetected, coupled together and amplified, a 4.4 to $5.0 \mathrm{GHz}$ tunable RF filter with a $30-\mathrm{MHz}$ bandwidth is used to select the desired oscillation mode.

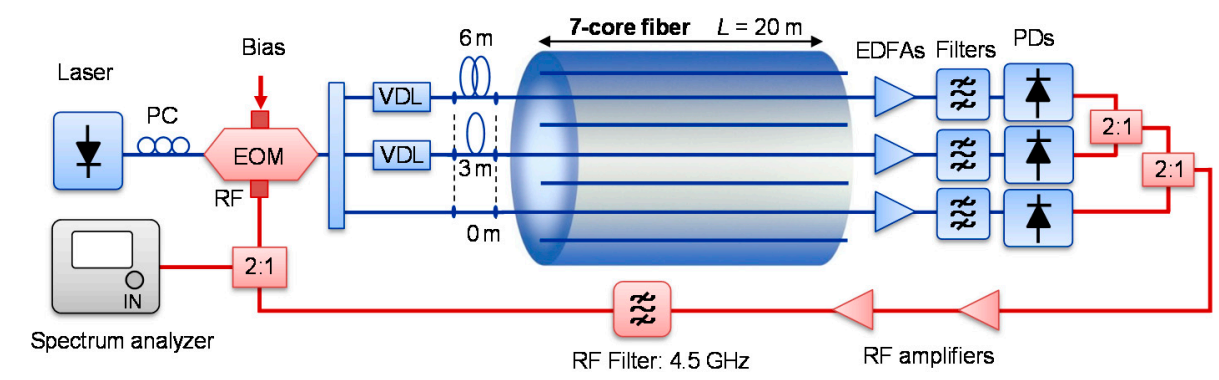

Figure 8. Setup for the experimental demonstration of a multi-cavity Vernier optoelectronic oscillator over a seven-core homogeneous fiber. PC: polarization controller; RF: radiofrequency; EOM: electro-optic modulator; VDL: variable delay line; EDFA: Erbium-doped fiber amplifier; VOA: variable optical attenuator; PD: photodetector (blue: optical path; red: electrical path) [59].

In general, the oscillation frequencies of a multi-cavity OEO verify $f_{o}=n_{i} / \tau_{i}$, for $n_{i}=1,2, \ldots$, $(i=1,2, \ldots, N)$, where $N$ is the total number of cavities and $\tau_{i}$ is the time delay experienced in each cavity $i$ including both the optical and the RF delays. The oscillation frequencies for the Vernier configuration verify $f_{o}=n / \Delta \tau$ (for $n=1,2, \ldots$ ) where $\Delta \tau$ is the incremental delay between cavities that produces the Vernier effect [59]. Figure 9a shows the measured RF spectrum for the three-cavity Vernier OEO where we matched the oscillating modes of all three cavities at the frequency located near $4.494 \mathrm{GHz}$. For the sake of comparison, we have also plotted the resulting spectrum for a two-cavity Vernier configuration over the same fiber. A 10-dB power reduction is observed at the spurious peaks corresponding to the minor oscillating modes of the single cavities when going from two to three loops. Figure $9 \mathrm{~b}$ gathers three consecutive oscillating modes for the 3-loop configuration featuring a FSR of $61 \mathrm{MHz}$. We can see that the 30-MHz RF filter bandwidth is then narrow enough to allow single-mode 
oscillation for the Vernier configuration. Regarding the frequency stability of the OEO, we observed that the oscillating mode could be shifted a few $\mathrm{kHz}$ due to the lack of proper isolation and, probably, due to possible fluctuations of the pump current applied to the EDFAs. This could actually induce non-desired mode-hopping effects. Nevertheless, the short lengths that characterized the fiber loops contribute to minimize this effect since they result in single-cavity FSRs (when each cavity operates in isolation) on the order of $2 \mathrm{MHz}$. Even more important, we observed that this effect was strongly reduced as we increased the number of cavities from two to three, so we can consider our three-cavity Vernier architecture considerably robust against possible mode hopping effects.
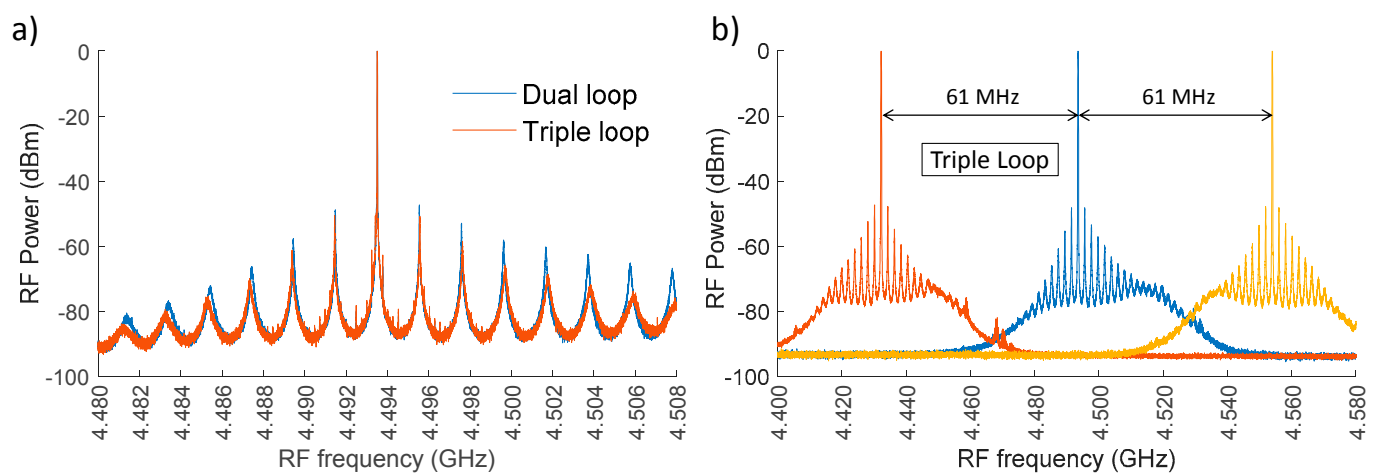

Figure 9. Experimental oscillation spectra of a multi-cavity Vernier optoelectronic oscillator using a 20-m seven-core fiber for: (a) dual-versus triple-loop operation; and (b) three consecutive oscillation modes for the triple-loop operation [59].

Figure 10 shows the measured phase noise spectrum for the three-cavity Vernier architecture (compared to the two-cavity Vernier architecture), where we observe a $-85 \mathrm{dBc} / \mathrm{Hz}$ phase noise level at a $10-\mathrm{KHz}$ offset from the carrier that decreases down to $-130 \mathrm{dBc} / \mathrm{Hz}$ for a $1-\mathrm{MHz}$ offset. We must note that the phase noise values can be significantly reduced by using low-residual phase noise components, by reducing the optical losses and thus suppressing the need for optical amplifiers, and by increasing the MCF length.

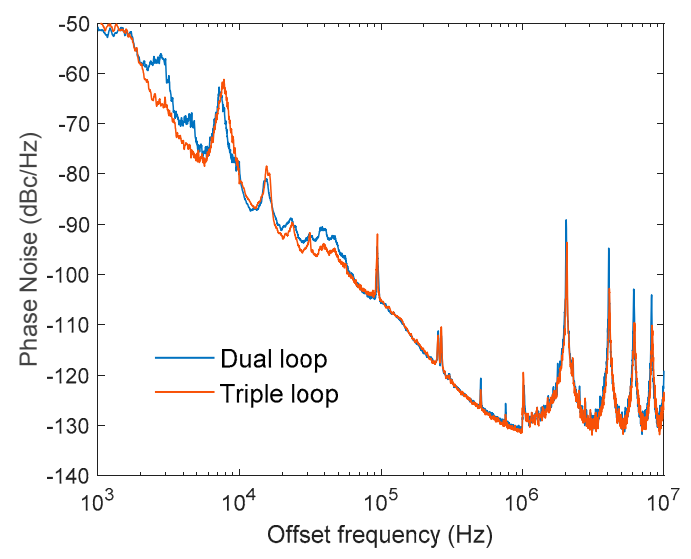

Figure 10. Experimental phase noise spectrum of a multi-cavity optoelectronic oscillator using a 20-m seven-core fiber comparing dual- and triple-loop Vernier configurations [59].

In addition, as we proposed for 2D tunable true time delay operation, dispersion-engineered heterogeneous MCFs can be applied to implement tunable OEO operation. In [55], we introduced vernier OEO operation built upon heterogeneous MCF links with long-cavity lengths $(>1 \mathrm{~km})$ that can provide high-spectral purity and multi-GHz oscillation mode spectral separation, while providing lower phase noise operation. If the dispersion parameter $D$ in each core is a multiple of a basic value 
$\Delta D$ (incremental dispersion parameter), the oscillation frequency for a core with a length $L$ is given by [55]:

$$
f_{o}=m /\left(\tau_{g o} L+\Delta D L \Delta \lambda\right), \text { for } m=1,2, \ldots
$$

where $\tau_{g o}$ is the group delay per unit of length at a given anchor wavelength $\lambda_{o}$ and $\Delta \lambda=\lambda-\lambda_{o}$ A distinctive feature of this OEO is the possibility of tuning the oscillation frequency by changing $\Delta \lambda$, in other words, by using a tunable laser. Figure 11 shows this relationship (Figure 11, left) and the oscillation frequencies (Figure 11, right) in between 10 and $20 \mathrm{GHz}$, computed for a set of selected values of $\Delta \lambda$.
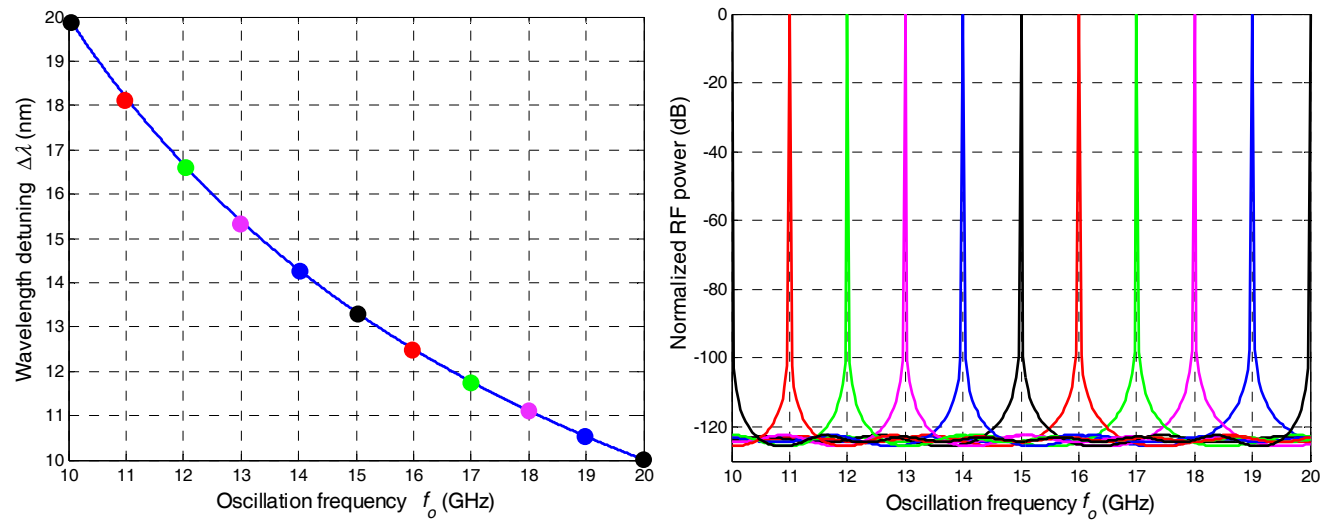

Figure 11. (Left) Oscillation frequency versus wavelength detuning for a vernier multi-cavity optoelectronic oscillator based on a seven-core heterogeneous fiber for $\tau_{g o}=5 \mathrm{~ns} / \mathrm{m}, D=1 \mathrm{ps} /(\mathrm{km} \cdot \mathrm{nm})$ and $L=5 \mathrm{~km}$; and (Right) corresponding oscillation spectrum for the different wavelength detuning positions [55].

\section{Conclusions}

We have reviewed the application of space-division multiplexing optical technologies for Microwave Photonics signal processing based on the use of both homogeneous and heterogeneous multicore fibers. In the case of heterogeneous multicore fibers, we showed that the proper design of the trench-assisted refractive index profile of each core results in length-distributed true time delay lines (up to a few $\mathrm{km}$ ) with a linear tunability with the operational optical wavelength. In the case of homogeneous multicore fibers, the selective incorporation of FBGs placed at different positions along the individual cores of the fiber allows the implementation of compact delay line devices with a length lower than $10 \mathrm{~cm}$. We like to name these solutions as fiber-distributed signal processing elements since they can process the signal while it is being distributed, for instance, in the context of $5 \mathrm{G}$ radio access networks. In this framework, multiple functionalities can be accomplished, including arbitrary waveform generation, reconfigurable signal filtering, optical beamforming networks for phased array antennas and analogue-to-digital conversion. A particular functionality that has been gathered in this paper is multicavity optoelectronic oscillation, where the required optical loops can be provided by the different cores of a single MCF in a variety of configurations. Future fiber-wireless access networks will benefit from the reported SDM approach in terms of compactness as compared to a set of parallel single-mode fibers, performance stability against mechanical or environmental conditions and operation versatility offered by the simultaneous use of the spatial- and wavelength-diversity domains. Moreover, the synergistic combination of photonic integrated circuits (understood as a "vertical integration") and the SDM fiber technologies gathered in this work (understood as a "horizontal integration") will contribute to the reduction of size, weight and power consumption that will drive the future of Microwave Photonics.

The research work reviewed here opens the way towards the development of parallel fiber-based signal processing systems for microwave and millimeter-wave signals that will be applicable to a myriad of fields including physical, chemical and smart structure sensing, medical imaging, optical coherence tomography, as well as broadband electronic and RF measurement instrumentation. Further research 
on this topic will be accomplished mainly in the framework of the European Consolidator Grant InnoSpace awarded by the European Research Council. In this sense, we will address among others: (1) the fabrication and subsequent experimental demonstration of dispersion-engineered heterogeneous MCFs for tunable time delay operation; (2) how to scale the number of cores beyond seven in both heterogeneous MCF link solutions and FBG-inscribed MCF devices; (3) the experimental demonstration of OEOs built upon heterogeneous MCFs; (4) the improvement of the phase noise performance in general in MCF-based OEOs; and (5) the consideration of other space-division multiplexing technologies for microwave photonics signal processing.

Acknowledgments: This research was supported by the ERC Consolidator Grant 724663; the Spanish Projects TEC2015-62520-ERC, TEC2014-60378-C2-1-R and TEC2016-80150-R; the Valencian Research Excellency Award Program GVA PROMETEO 2013/012; the Spanish MECD FPU Scholarship (FPU13/04675) for J. Hervás; the Spanish scholarships MINECO BES-2015-073359 for S. García; and the Spanish MINECO Ramon y Cajal program RYC-2014-16247 for I. Gasulla.

Author Contributions: I.G. conceived the ideas and supervised the work. S.G. and I.G. worked on the design and evaluation of delay lines based on heterogeneous MCFs. J.H., D.B. and S.S. were involved in the fabrication and experimental characterization of delay lines based on the inscription of FBGs in homogeneous MCFs. S.G. and I.G. worked on the optoelectronic oscillators. S.G. and I.G. wrote the manuscript.

Conflicts of Interest: The authors declare no conflict of interest.

\section{References}

1. Richardson, D.J.; Fini, J.M.; Nelson, L.E. Space-division multiplexing in optical fibres. Nat. Photonics 2013, 7, 354-362. [CrossRef]

2. Puttnam, B.J.; Luís, R.S.; Klaus, W.; Sakaguchi, J.; Mendinueta, J.M.D.; Awaji, Y.; Wada, N.; Tamura, Y.; Hayashi, T.; Hirano, M.; et al. $2.15 \mathrm{~Pb} / \mathrm{s}$ transmission using a 22 core homogeneous single-mode multi-core fiber and wideband optical comb. In Proceedings of the 2015 41st European Conference on Optical Communications (ECOC), Valencia, Spain, 27 September-1 October 2015.

3. Koshiba, M. Design aspects of multicore optical fibers for high-capacity long-haul transmission. In Proceedings of the IEEE 2014 International Topical Meeting on Microwave Photonics and the 2014 9th Asia-Pacific Microwave Photonics Conference (APMP), Sendai, Japan, 20-23 October 2014; pp. 318-323.

4. Hayashi, T.; Sasaki, T.; Sasaoka, E.; Saitoh, K.; Koshiba, M. Physical interpretation of intercore crosstalk in multicore fiber: Effects of macrobend, structure fluctuation and microbend. Opt. Express 2013, 21, 5401-5412. [CrossRef] [PubMed]

5. Tu, J.; Saitoh, K.; Koshiba, M.; Takenaga, K.; Matsuo, S. Optimized design method for bend-insensitive heterogeneous trench-assisted multi-core fiber with ultra-low crosstalk and high core density. J. Lightw. Technol. 2013, 31, 2590-2598.

6. Galvé, J.M.; Gasulla, I.; Sales, S.; Capmany, J. Reconfigurable Radio Access Networks using Multicore Fibers. IEEE J. Quantum Electron. 2016, 52, 1-7. [CrossRef]

7. Saitoh, K.; Matsuo, S. Multicore fiber technology. J. Lightw. Technol. 2016, 34, 55-66. [CrossRef]

8. Matsuo, S.; Takenaga, K.; Sasaki, Y.; Amma, Y.; Saito, S.; Saitoh, K.; Matsui, T.; Nakajima, K.; Mizuno, T.; Takara, H.; et al. High-spatial-multiplicity multicore fibers for future dense space-division-multiplexing systems. J. Lightw. Technol. 2016, 34, 1464-1475. [CrossRef]

9. Koshiba, M.; Saitoh, K.; Kokubun, Y. Heterogeneous multi-core fibers: Proposal and design principle. IEICE Electron. Express 2009, 6, 98-103. [CrossRef]

10. Tu, J.; Long, K.; Saitoh, K. An efficient core selection method for heterogeneous trench-assisted multi-core fiber. IEEE Photonics Technol. Lett. 2016, 28, 810-813. [CrossRef]

11. Feng, Z.; Xu, L.; Wu, Q.; Tang, M.; Fu, S.; Tong, W.; Shum, P.P.; Liu, D. Ultra-high capacity WDM-SDM optical access network with self-homodyne detection downstream and 32QAM-FBMC upstream. Opt. Express 2017, 25, 5951-5961. [CrossRef] [PubMed]

12. Ye, F.; Tu, J.; Saitoh, K.; Morioka, T. Simple analytical expression for crosstalk estimation in homogeneous trench-assisted multi-core fibers. Opt. Express 2014, 22, 23007-23018. [CrossRef] [PubMed]

13. Masanori, K.; Saitoh, K.; Takenaga, K.; Matsuo, S. Analytical expression of average power-coupling coefficients for estimating intercore crosstalk in multicore fibers. IEEE Photonics J. 2012, 4, 1987-1995. 
14. Zhou, J. A non-orthogonal coupled mode theory for super-modes inside multi-core fibers. Opt. Express 2014, 22, 10815-10824. [CrossRef] [PubMed]

15. Diamandi, H.H.; London, Y.; Zadok, A. Opto-mechanical inter-core cross-talk in multi-core fibers. Optica 2017, 4, 289-297. [CrossRef]

16. Koshiba, M.; Saitoh, K.; Takenaga, K.; Matsuo, S. Multi-core fiber design and analysis: Coupled-mode theory and coupled-power theory. Opt. Express 2011, 19, B102-B111. [CrossRef] [PubMed]

17. Matsuo, S. Crosstalk behavior of cores in multi-core fiber under bent condition. IEICE Electron. Express 2011, 8, 385-390. [CrossRef]

18. Hayashi, T.; Nagashima, T.; Shimakawa, O.; Sasaki, T.; Sasaoka, E. Crosstalk variation of multi-core fibre due to fibre bend. In Proceedings of the 36th European Conference on Optical Communications (ECOC), Torino, Italy, 19-23 September 2010.

19. Fini, J.M.; Zhu, B.; Taunay, T.F.; Yan, M.F. Statistics of crosstalk in bent multicore fibers. Opt. Express 2010, 18, 15122-15129. [CrossRef] [PubMed]

20. Hayashi, T.; Taru, T.; Shimakawa, O.; Sasaki, T.; Sasaoka, E. Design and fabrication of ultra-low crosstalk and low-loss multi-core fiber. Opt. Express 2011, 19, 16576-16592. [CrossRef] [PubMed]

21. Chan, F.; Lau, A.P.T.; Tam, H.Y. Mode coupling dynamics and communication strategies for multi-core fiber systems. Opt. Express 2012, 20, 4548-4563. [CrossRef] [PubMed]

22. Chan, F.; Mudhana, G.; Lee, B. Influence of dissimilar cores on the mode-coupling dynamics of multicore fibers. IEEE Photonics Technol. Lett. 2012, 24, 1399-1401. [CrossRef]

23. Fini, J.; Zhu, B.; Taunay, T.F.; Yan, M.F.; Abedin, K.S. Crosstalk in multicore fibers with randomness: Gradual drift vs. short-length variations. Opt. Express 2012, 20, 949-959. [CrossRef] [PubMed]

24. Macho, A.; Morant, M.; Llorente, R. Experimental evaluation of nonlinear crosstalk in multi-core fiber. Opt. Express 2015, 23, 18712-18718. [CrossRef] [PubMed]

25. Sasaki, Y.; Takenaga, K.; Aikawa, K.; Miyamoto, Y.; Morioka, T. Single-mode 37-core fiber with a cladding diameter of $248 \mu \mathrm{m}$. In Proceedings of the Optical Fiber Communication Conference (OFC), Los Angeles, CA, USA, 19-23 March 2017.

26. Amma, Y.; Sasaki, Y.; Takenaga, K.; Matsuo, S.; Tu, J.; Saitoh, K.; Koshiba, M.; Morioka, T.; Miyamoto, Y. High-density multicore fiber with heterogeneous core arrangement. In Proceedings of the Optical Fiber Communication Conference (OFC) 2015, Los Angeles, CA, USA, 22-26 March 2015.

27. Ryf, R.; Chen, H.; Fontaine, N.K.; Velazquez-Benitez, A.M.; Antonio-Lopez, J.; Jin, C.; Huang, B.; Bigot-Astruc, M.; Molin, D.; Achten, F.; et al. 10-Mode Mode-Multiplexed Transmission over 125-km Single-Span Multimode Fiber. In Proceedings of the 41st European Conference on Optical Communications (ECOC), Valencia, Spain, 27 September-1 October 2015.

28. Sakaguchi, J.; Klaus, W.; Mendinueta, J.M.; Puttnam, B.J.; Luis, R.S.; Awaji, Y.; Wada, N.; Hayashi, T.; Nakanishi, T.; Watanabe, T.; et al. Realizing a 36-core, 3-mode Fiber with 108 Spatial Channels. In Proceedings of the Optical Fiber Communication Conference (OFC), Los Angeles, CA, USA, 22-26 March 2015.

29. Doerr, C.R. Silicon photonics for space-division multiplexing. In Proceedings of the IEEE Photonics Society Summer Topical Meeting, Seattle, WA, USA, 9-11 July 2012; pp. 242-243.

30. Koonen, A.M.J.; Chen, H.-S.; van den Boom, H.P.A.; Raz, O. Silicon Photonic Integrated Mode Multiplexer. In Proceedings of the 2012 IEEE Photonics Society Summer Topical Meeting, Seattle, WA, USA, 9-11 July 2012; pp. 240-241.

31. Ding, Y.; Kamchevska, V.; Dalgaard, K.; Bacco, D.; Rottwitt, K.; Hu, H.; Galili, M.; Morioka, T.; Oxenløwe, L.K. Silicon photonics for multicore fiber communication. In Proceedings of the Asia Communications and Photonics Conference, Wuhan, China, 2-5 November 2016.

32. Lindenmann, N.; Dottermusch, S.; Goedecke, M.L.; Hoose, T.; Billah, M.R.; Onanuga, T.P.; Hofmann, A.; Freude, W.; Koos, C. Connecting Silicon Photonic Circuits to Multicore Fibers by Photonic Wire Bonding. IEEE J. Lightw. Technol. 2016, 33, 755-760. [CrossRef]

33. Samsung Electronics Co, “5G Vision”. Available online: http://www.samsung.com/global/businessimages/insights/2015/Samsung-5G-Vision-0.pdf (accessed on 15 March 2015).

34. Waterhouse, R.; Novak, D. Realizing 5G: Microwave Photonics for 5G Mobile Wireless Systems. IEEE Microw. Mag. 2015, 16, 84-92. [CrossRef]

35. Barrera, D.; Gasulla, I.; Sales, S. Multipoint two-dimensional curvature optical fiber sensor based on a non-twisted homogeneous four-core fiber. IEEE J. Lightw. Technol. 2015, 33, 2445-2450. [CrossRef] 
36. Capmany, J.; Mora, J.; Gasulla, I.; Sancho, J.; Lloret, J.; Sales, S. Microwave photonic signal processing. IEEE J. Lightw. Technol. 2013, 31, 571-586. [CrossRef]

37. Seeds, A. Microwave photonics. IEEE Trans. Microw. Theory Tech. 2002, 50, 877-887. [CrossRef]

38. Capmany, J.; Novak, D. Microwave photonics combines two worlds. Nat. Photonics 2007, 1, 319-330. [CrossRef]

39. Yao, J. Microwave photonics. IEEE J. Lightw. Technol. 2009, 27, 314-335. [CrossRef]

40. Wilner, K.; Van Den Heuvel, A.P. Fiber-optic delay lines for microwave signal processing. Proc. IEEE 1976, 64, 805-807. [CrossRef]

41. Capmany, J.; Pastor, D.; Ortega, B. New and flexible fiber-optic delay-line filters using chirped Bragg gratings and laser arrays. IEEE Trans. Microw. Theory Tech. 1999, 47, 1321-1326. [CrossRef]

42. Zeng, F.; Yao, J. All-optical microwave filters using uniform fiber Bragg gratings with identical reflectivities. IEEE J. Lightw. Technol. 2005, 23, 1410-1418. [CrossRef]

43. Wang, C.; Yao, J. Fiber Bragg gratings for microwave photonics subsystems. Opt. Express 2013, 21, 22868-22884. [CrossRef] [PubMed]

44. Morton, P.A.; Khurgin, J.B. Microwave photonic delay line with separate tuning of the optical carrier. IEEE Photonics Technol. Lett. 2009, 21, 1686-1688. [CrossRef]

45. Marpaung, D.; Roeloffzen, C.; Heideman, R.; Leinse, A.; Sales, S.; Capmany, J. Integrated microwave photonics. Lasers Photonics Rev. 2013, 7, 506-538. [CrossRef]

46. Sancho, J.; Bourderionnet, J.; Lloret, J.; Combrié, S.; Gasulla, I.; Xavier, S.; Sales, S.; Colman, P.; Lehoucq, G.; Dolfi, D.; et al. Integrable microwave filter based on a photonic crystal delay line. Nat. Commun. 2012, 3, 1075. [CrossRef] [PubMed]

47. Ohman, F.; Yvind, K.; Mørk, J. Slow Light in a Semiconductor Waveguide for True-Time Delay Applications in Microwave Photonics. IEEE Photonics Technol. Lett. 2007, 19, 1145-1157. [CrossRef]

48. Gasulla, I.; Capmany, J. Microwave photonics applications of multicore fibers. IEEE Photonics J. 2012, 4, 877-887. [CrossRef]

49. Garcia, S.; Gasulla, I. Design of Heterogeneous Multicore Fibers as Sampled True-Time Delay Lines. Opt. Lett. 2015, 40, 621-624. [CrossRef] [PubMed]

50. Garcia, S.; Gasulla, I. Dispersion-engineered multicore fibers for distributed radiofrequency signal processing. Opt. Express 2016, 24, 20641-20654. [CrossRef] [PubMed]

51. Gasulla, I.; Barrera, D.; Hervás, J.; Sales, S. Spatial Division Multiplexed Microwave Signal processing by selective grating inscription in homogeneous multicore fibers. Sci. Rep. 2017, 7, 41727. [CrossRef] [PubMed]

52. Birks, T.; Mangan, B.; Diez, A.; Cruz, J.L.; Leon-Saval, S.; Bland-Hawthorn, J.; Murphy, D. Multicore optical fibres for astrophotonics. In Proceedings of the CLEO/Europe and EQEC 2011 Conference Digest, Munich, Germany, 22-26 May 2011; paper JSIII2_1.

53. Yao, X.S.; Maleki, L. Multiloop optoelectronic oscillator. IEEE J. Quantum Electron. 2000, 36, 79-84. [CrossRef]

54. Bánky, T.; Horváth, B.; Berceli, T. Optimum configuration of multiloop optoelectronic oscillators. J. Opt. Soc. Am. B 2006, 23, 1371-1380. [CrossRef]

55. Garcia, S.; Gasulla, I. Multi-cavity optoelectronic oscillators using multicore fibers. Opt. Express 2015, 23, 2403-2415. [CrossRef] [PubMed]

56. Li, W.; Yao, J. An optically tunable optoelectronic oscillator. J. Lightwave Technol. 2010, 28, $2640-2645$. [CrossRef]

57. Lelièvre, O.; Crozatier, V.; Baili, G.; Berger, P.; Pillet, G.; Dolfi, D.; Morvan, L.; Goldfarb, F.; Bretenaker, F.; Llopis, O. Ultra-low phase noise $10 \mathrm{GHz}$ dual loop optoelectronic oscillator. In Proceedings of the IEEE 2016 International Topical Meeting on Microwave Photonics, Long Beach, CA, USA, 31 October-3 November 2016; pp. 106-109.

58. Yao, X.S.; Maleki, L. Optoelectronic microwave oscillator. J. Opt. Soc. Am. B 1996, 13, 1725-1735. [CrossRef]

59. Garcia, S.; Gasulla, I. Experimental demonstration of multi-cavity optoelectronic oscillation over a multicore fiber. Opt. Express 2017, 25, 23663-23668. [CrossRef] [PubMed]

(C) 2017 by the authors. Licensee MDPI, Basel, Switzerland. This article is an open access article distributed under the terms and conditions of the Creative Commons Attribution (CC BY) license (http:/ / creativecommons.org/licenses/by/4.0/). 